\title{
ON THE THEORY OF THE PLASTIC POTENTIAL*
}

\author{
BY HANS ZIEGLER, Zürich
}

1. Introduction. The theory of the plastic potential, proposed by v. Mises [1], connects the yield condition and the flow rule of a plastic solid. In its generalized form established by Prager [2], the theory may be stated as follows:

Let $q_{k}(k=1,2, \cdots, n)$ be the generalized strains, i.e. a set of coordinates specifying the deformation of the plastic body in the sense of analytical mechanics, and let $q_{k}^{*}$, $q_{k}^{p}$ denote respectively the elastic and the plastic components of $q_{k}$. If the work done on an infinitesimal increment of strain is given by

$$
d A=Q_{k} d q_{k},
$$

where the summation convention has been applied, the $Q_{k}$ are the generalized forces in the sense of analytical mechanics or, according to Prager, the generalized stresses corresponding to the coordinates $q_{k}$.

The generalized states of strain and stress may be depicted by points with position vectors q, $\mathbf{Q}$ in Euclidean $n$-space $R_{n}$, or by the vectors $\mathrm{q}, \mathbf{Q}$ themselves. Defining the scalar product of two vectors in $R_{n}$ by

$$
\mathbf{Q} \cdot \mathbf{q}=Q_{k} q_{k},
$$

we obtain for the work (1.1) the representation

$$
d A=\mathbf{Q} \cdot d \mathbf{q} .
$$

Consider the solid in an arbitrary stage of the deformation process, and let $\mathbf{Q}$ denote the actual state of stress. Any state of stress $Q^{*}$ which can be reached from $Q$ without plastic flow will be called nonplastic. All of the $\mathbf{Q}^{*}$ constitute the nonplastic domain $\bar{R}_{n}$ in $R_{n}$, and any stress increment of the type $\mathbf{Q}^{*}-\mathbf{Q}$ will be denoted as nonplastic ${ }^{1}$ ). The yield limit is a hypersurface in $R_{n}$, defined by those nonplastic states of stress the infinitesimal changes of which are not exclusively nonplastic.

The theory of the plastic potential firstly stipulates that the elastic strains $q^{\circ}$ follow from the stresses $Q$ according to the laws of elasticity. The plastic strain increment $d \mathbf{q}^{p}$ corresponding to given values of the stress $\mathbf{Q}$ and the stress increment $d \mathbf{Q}$ is zero for any state of stress lying in the nonplastic domain but not at the yield limit. For states of stress at the yield limit, $d \mathbf{q}^{p}$ may be different from zero; it is secondly stipulated that

$$
\left(\mathbf{Q}^{*}-\mathbf{Q}\right) \cdot d \mathbf{q}^{p} \leq 0
$$

for any nonplastic stress increment $\mathbf{Q}^{*}-\mathbf{Q}$, and that

$$
d \mathbf{Q} \cdot d \mathbf{q}^{p} \geq 0 .
$$

In virtue of (1.4) the nonplastic domain $\bar{R}_{n}$ is convex.

*Received June 17, 1960.

'In Prager's or Koiter's terminology [3], $\bar{R}_{n}$ is the elastic domain and $\mathrm{Q}^{*}$ an allowable state of stress. The notations used here emphasize the fact that ideally plastic as well as hardening solids are considered. 
2. Formulation of problem. So far, the theory of the plastic potential is but a hypothesis. This has been emphasized already by v. Mises; besides, his version was restricted to a volume element with strains $\epsilon_{i j}$ and stresses $\sigma_{i j}$. The generalization of Section 1 is due to Prager; the special form (1.4), (1.5) does not refer to a potential function and was given by Drucker [4].

Several authors have tried to provide a basis for the theory (see [3], p. 180). Bishop and Hill [5] have shown that (1.4) follows for the element of a polycrystalline aggregate from plausible assumptions concerning the behaviour of the single crystals. Drucker [4] has based the theory on a postulate regarding the work done on a prestressed element by an external agency in a cycle of application and removal. The author [6] has suggested a generalization of Onsager's theory of irreversible processes [7] to nonlinear cases, thus providing a thermodynamic basis for the theory of the plastic potential.

Either one of these approaches is based in its turn on certain postulates some of which are open to criticism [8]. Thus, it seemed worthwhile for once to reject any kind of postulate and to limit the scope of the investigation to a purely mathematical proof for the theory in Prager's generalized form, based on the sole assumption that it holds in v. Mises' sense for an element of volume. This proof has been provided by the author, in [8] for rigid-plastic materials and in [9] for elastic-plastic solids. The present paper gives a condensed version of the two articles and at the same time provides a simplification of the proof.

3. The rigid-plastic element. In a rigid-plastic solid, $\mathbf{q}^{e}=0$ and hence $q=q^{p}$. The local states of strain and stress are given by $\epsilon_{i j}, \sigma_{i j}$ respectively and may be depicted by the vectors $\mathrm{e}, \mathrm{s}$ in Euclidean 9 -space $R_{9}$. Defining the scalar product in $R_{9}$ by

$$
\mathbf{s} \cdot \mathbf{e}=\sigma_{i j} \epsilon_{i i},
$$

we obtain for the work per unit volume done on an infinitesimal increment of strain

$$
d \bar{A}=\sigma_{i j} d \epsilon_{i j}=\mathbf{s} \cdot d \mathbf{e} .
$$

In virtue of the symmetry of the strain and stress tensors, the vectors e and $\mathbf{s}$ actually lie in a linear subspace $R_{6}$ of $R_{9}$. Here, the nonplastic domain may be defined according to the rules of Section 1 . If we assume that the theory of the plastic potential is valid in v. Mises' sense, it follows from (1.4) and (1.5) that

$$
\left(\mathbf{s}^{*}-\mathbf{s}\right) \cdot d \mathbf{e}=\left(\sigma_{i j}^{*}-\sigma_{i i}\right) d \epsilon_{i j} \leq 0
$$

for any nonplastic stress increment $\mathbf{s}^{*}-\mathbf{s}$, and that

$$
d \mathbf{s} \cdot d \mathbf{e}=d \sigma_{i j} d \epsilon_{i j} \geq 0 .
$$

These relations also hold (with the equality sign) for states of stress below the yield limit. In virtue of (3.3) the nonplastic domain is convex.

4. The rigid-plastic solid. The states of strain and stress of the whole body $B$ are given by the functions $\epsilon_{i j}\left(x_{k}\right), \sigma_{i j}\left(x_{k}\right)$ respectively, either one of them depending on the coordinates $x_{k}$. These functions may be represented by vectors $\mathrm{E}, \mathrm{S}$ in function space $F$. Let the scalar product in $F$ be defined by the volume integral

$$
\mathbf{S} \cdot \mathbf{E}=\int_{B} \sigma_{i j} \epsilon_{i j} d V
$$

extended over the entire body $B$. This definition is admissible (see [10]), since it satisfies the commutative law, $\mathbf{S} \cdot \mathbf{E}=\mathbf{E} \cdot \mathbf{S}$, the distributive law, $\mathbf{S} \cdot\left(\mathbf{E}+\mathbf{E}^{\prime}\right)=\mathbf{S} \cdot \mathbf{E}+\mathbf{S} \cdot \mathbf{E}^{\prime}$, the 
rule $\mathbf{S} \cdot \mathbf{O}=\mathbf{0}$ for multiplication by zero and the associative law for scalars, $(a \mathbf{S}) \cdot \mathbf{E}=a$ (S.E). Besides, the definition provides $F$ with a positive definite metric. On account of (4.1), the work done in an infinitesimal increment of strain is

$$
d A=\int_{B} \sigma_{i j} d \epsilon_{i j} d V=\mathbf{S} \cdot d \mathbf{E} .
$$

The representation considered here is not restricted to kinematically admissible states of strain nor to statically admissible states of stress; it holds for any states for which the integrals in (4.1), (4.2) exist. The nonplastic domain $\bar{F}$ in $F$ may again be defined according to the rules of Section 1. In general, certain elements of $B$ reach their local yield limit for states of stress $\mathbf{S}$ still inside the yield limit of the whole body. Plastic flow sets in when a sufficiently large domain of $B$ has become plastic. At this stage, the state of stress in any element of $B$ either lies below or on its local yield limit. Since none of the elements undergoes plastic flow in a nonplastic stress increment of the entire body, it follows from (3.3) that

$$
\left(\mathrm{S}^{*}-\mathrm{S}\right) \cdot d \mathbf{E}=\int_{B}\left(\sigma_{i j}^{*}-\sigma_{i i}\right) d \epsilon_{i j} d V \leq 0
$$

for any nonplastic stress increment $\mathbf{S}^{*}-\mathbf{S}$. Also, on account of (3.4),

$$
d \mathbf{S} \cdot d \mathbf{E}=\int_{B} d \sigma_{i j} d \epsilon_{i j} d V \geq 0 .
$$

Hence, the theory of the plastic potential, if valid for the element, likewise applies to the body as a whole. Incidentally, relations (4.3) and (4.4) also hold (with equality sign) for states of stress below the yield limit. In virtue of (4.3), also the nonplastic domain $\bar{F}$ is convex.

5. Generalized strains and stresses. Section 4 provides a basis for the rigorous treatment of a rigid-plastic body. In numerous cases, however, one is compelled to simplify the problem by introducing generalized strains and stresses as defined in Section 1. This means necessarily that only states of strain are considered which can be described by a finite set of parameters $q_{k}(k=1,2, \cdots, n)$. Such a reduction of the degree of freedom can be realized by introducing additional constraints; in certain cases also the elimination of originally existing constraints may result in a simplification (see examples in [8] and [9]). It is obvious that this process is only justified as long as it does not appreciably modify the actual state of strain.

The limitation considered here implies that only states of strain $\mathbf{E}$ are considered which belong to a certain subspace $F_{n}$ of $F$. It does not involve a similar restriction with respect to the states of stress. Let $\mathbf{E}^{(k)}$ denote the state of strain in function space $F$ corresponding to the generalized strain $q_{k}=1, q_{i}=0(i \neq k)$. The subspace $F_{n}$ then is defined by the states of strain

$$
\mathbf{E}=q_{k} \mathbf{E}^{(k)} .
$$

It is reasonable to assume that the vectors $\mathbf{E}^{(k)}$ are linearly independent. In this event, there is a one-to-one correspondence between the vectors $\mathrm{E}$ in $F_{n}$ and $\mathrm{q}$ in $R_{n}$. On account of (4.2), (5.1) and (1.1), the work done by the stress $S$ on an infinitesimal strain increment $d \mathrm{E}$ belonging to $F_{n}$ is

$$
d A=\mathbf{S} \cdot d \mathbf{E}=\mathbf{S} \cdot \mathbf{E}^{(k)} d q_{k}=Q_{k} d q_{k} .
$$


Hence, the generalized stresses are given by the scalar products

$$
Q_{k}=\mathbf{S} \cdot \mathbf{E}^{(k)} \text {. }
$$

It follows immediately that any state of stress $\mathrm{S}$ in $F$ is represented in $R_{n}$ by a unique vector $\mathbf{Q}$ which, conversely, is the image of an infinity of vectors $\mathbf{S}$.

The straight section connecting the points in $F$ with position vectors $\mathbf{S}^{\prime}, \mathbf{S}^{\prime \prime}$ is given by

$$
\mathbf{S}=\mathbf{S}^{\prime}+a\left(\mathbf{S}^{\prime \prime}-\mathbf{S}^{\prime}\right), \quad(0 \leq a \leq 1) .
$$

If $Q, Q^{\prime}, Q^{\prime \prime}$ are the vectors in $R_{n}$ corresponding to $S, S^{\prime}, S^{\prime \prime}$, we obtain from (5.3) and (5.4)

$$
Q_{k}=Q_{k}^{\prime}+a\left(Q_{k}^{\prime \prime}-Q_{k}^{\prime}\right), \quad(0 \leq a \leq 1) .
$$

Thus, the image in $R_{n}$ of the section (5.4) is the straight section connecting the points with position vectors $\mathbf{Q}^{\prime}, \mathbf{Q}^{\prime \prime}$.

Since the nonplastic domain $\bar{F}$ is convex, any vector $\mathbf{S}^{*}-\mathbf{S}$ representing a nonplastic stress increment lies entirely in $\bar{F}$. Its image in $R_{n}$ is the vector $\mathrm{Q}^{*}-\mathrm{Q}$ which, according to the definition of the nonplastic domain, lies entirely in $\bar{R}_{n}$. Conversely, any vector $\mathbf{Q}^{*}-\mathbf{Q}$ in $\bar{R}_{n}$ is the image of at least one vector $\mathbf{S}^{*}-\mathbf{S}$ connecting two points of $\bar{F}$. Since $\bar{F}$ is convex, $\mathrm{S}^{*}-\mathrm{S}$ lies entirely in $\bar{F}$; hence, any vector in $\bar{R}_{n}$ can be considered the image of a nonplastic stress increment.

From (5.2) and (4.3) we obtain

$$
\left(\mathbf{Q}^{*}-\mathbf{Q}\right) \cdot d \mathbf{q}=\left(\mathbf{S}^{*}-\mathbf{S}\right) \cdot d \mathbf{E} \leq \mathbf{0},
$$

where $\mathbf{Q}^{*}-\mathbf{Q}$ is an arbitrary nonplastic stress increment. Likewise, on account of (5.2) and (4.4),

$$
d \mathbf{Q} \cdot d \mathbf{q}=d \mathbf{S} \cdot d \mathbf{E} \geq \mathbf{0} .
$$

This is the proof that the theory of the plastic potential, if valid for the element, likewise applies to the treatment of the entire body in generalized strains and stresses. In virtue of (5.6), the nonplastic domain $\bar{R}_{n}$ is convex.

6. The elastic-plastic solid. In Section 1, no rules have been specified for the decomposition of the strain into its elastic and plastic components. In the case of a volume element, however, the decomposition is straightforward, provided the strains are sufficiently small ${ }^{2}$. Let us postulate that in the expression

$$
d \bar{A}=\mathbf{s} \cdot d \mathbf{e}^{e}+\mathbf{s} \cdot d \mathbf{e}^{p}
$$

following from (3.2) the first product represents the infinitesimal increase of elastic strain energy per unit volume, while the second one represents the work dissipated in the infinitesimal strain increment $d \mathbf{e}$. Then, $\mathbf{e}^{\boldsymbol{e}}$ is the strain corresponding to the stress $s$ according to the law of elasticity, and $e^{p}$ is the permanent strain still present after removal of the stress.

In order to define a similar decomposition for the finite body, let us postulate that also in

$$
d A=\mathbf{S} \cdot d \mathbf{E}^{e}+\mathbf{S} \cdot d \mathbf{E}^{p}
$$

${ }^{2}$ For a few critical observations, see [9]. 
the products are respectively the increment of elastic strain energy and the work of dissipation. Thus, $\mathbf{E}^{e}$ is built up from the local elastic strains $\mathbf{e}^{e}$ and $\mathbf{E}^{p}$ from the local plastic strains $\mathrm{e}^{p}$. Here, $\mathbf{E}^{e}$ may be different from the state of strain corresponding to the given loads if the body were elastic; also, $\mathbf{E}^{p}$ is not necessarily the state of strain after removal of the loads. On the other hand, the definition (6.2) implies that the work of dissipation is

$$
d \bar{A}^{p}=\mathbf{s} \cdot d \mathbf{e}^{p}=\sigma_{i j} d \epsilon_{i j}^{p}
$$

per unit volume and

$$
d A^{p}=\mathbf{S} \cdot d \mathbf{E}=\int_{B} \sigma_{i i} d \epsilon_{i i}^{p} d V
$$

for the entire body.

Let us now replace (3.2) by (6.3) and (4.2) by (6.4). Assuming that relations (3.3) and (3.4) hold for the plastic strain increment $d \mathrm{e}^{p}$ instead of $d \mathbf{e}$, and retracing the demonstration of Section 4, we arrive at (4.3) and (4.4) with ( $d \epsilon_{i j}^{p}$ instead of $d \epsilon_{i j}$ and) $d \mathrm{E}^{p}$ instead of $d \mathbf{E}$.

For the representation in generalized coordinates, the situation is similar. Let us postulate that the products in

$$
d A=\mathbf{Q} \cdot d \mathbf{q}^{\mathbf{e}}+\mathbf{Q} \cdot d \mathbf{q}^{\boldsymbol{D}}
$$

are respectively equal to the increment of elastic strain energy and the work of dissipation. Then (5.2) can be replaced by

$$
d A^{p}=\mathbf{S} \cdot d \mathbf{E}^{p}=\mathbf{Q} \cdot d \mathbf{q}^{p},
$$

and the remainder of Section 5 leads to (5.6) and (5.7) with ( $d \mathrm{E}^{p}$ instead of $d \mathrm{E}$ and) $d \mathbf{q}^{\nu}$ instead of $d \mathbf{q}$.

Thus, the results proved in Sections 4 and 5 also hold for the plastic strains in elasticplastic solids, provided the elastic and plastic strain components are defined by means of strain energy and dissipation work ${ }^{3}$.

7. Observations. In an elastic-plastic solid, the elements which reach their local yield limit are not surrounded by rigid material. Hence, plastic flow sets in as soon as the first elements become plastic. It follows that under otherwise identical circumstances the nonplastic domain of the elastic-plastic solid is usually distinct from the one of the rigid-plastic body. Moreover, any plastic flow is apt to modify the nonplastic domain. Hence, the yield limit of the elastic-plastic solid undergoes a continuous transformation as the plastic flow proceeds towards collapse. On the other hand, the yield limit of a non-hardening rigid-plastic solid is always the same.

In order to determine the nonplastic domain $\bar{R}_{n}$ in an arbitrary stage of the deformation process, it is necessary to consider the actual state of stress $\mathbf{S}$. From the generalized stresses $\mathbf{Q}$ alone no information concerning the yield limit is available. In the case of a non-hardening rigid-plastic body, the yield limit must be determined only once. Once it is known, the theory of the plastic potential may be applied in the sense of Section 1, and the problem can be treated henceforth in generalized strains and stresses. In fact, this is the reason why Prager's version of the theory offers an essential simpli-

sAs, e.g., in [6]. 
fication. In the case of an elastic-plastic solid, however, one is compelled to keep track of the continuous changes in shape of the yield limit. Since this requires that the actual state of stress $\mathbf{S}$ be pursued throughout the deformation process, the use of generalized strains and stresses does not seem to offer any advantages here, except, of course; in limit analysis.

\section{BibliograpHY}

[1] R. von Mises, Mechanik der plastischen Formänderung von Kristallen, Z. angew. Math. Mech. 8, 161 (1928).

[2] W. Prager, An'introduction to plasticity, Addison-Wesley, Reading, Mass. 1959, p. 13.

[3] W. T. Koiter, General theorems for elastic-plastic solids, in I. N. Sneddon and R. Hill, Progress in solid mechanics, North-Holland Publishing Co., Amsterdam 1960, p. 172.

[4] D. C. Drucker, Some implications of work hardening and ideal plasticity, Quart. Appl. Math. 7, 411 (1949).

[5] J. F. W. Bishop and R. Hill, $A$ theory of the plastic distortion of a polycrystalline aggregate under combined stresses, Phys. Mag. (7) 42, 414 (1951).

[6] H. Ziegler, An attempt to generalize Onsager's principle, and its significance for rheological problems, Z. angew. Math. Phys. 9b, 748 (1958).

[7] L. Onsager, Reciprocal relations in irreversible processes, Phys. Rev. 37 (II), 405 (1931) and 38 (II), 2265 (1931).

[8] H. Ziegler, Ueber den Zusammenhang zwischen der Fliessbedingung eines starrplastischen Körpers und seinem Fliessgesetz, Z. angew. Math. Phys. 11, 413 (1960).

[9] H. Ziegler, Ueber den Zusammenhang zwischen der Fliessbedingung eines elastisch-plastischen Körpers und seinem Fliessgesetz, Z. angew. Math. Phys. 12, (1961).

[10] J. L. Synge, The hypercircle in mathematical physics, Cambridge University Press 1957, p. 37. 